\section{Contribution of branching order of dendrites to morphology of neural cells}

\author{
Mina Elias, Noha M. Salem, Manal M. Awad \\ and Medhat A. ElMessiery*
}

Department of Engineering Mathematics and Physics, Faculty of Engineering, Cairo University, Giza, 12613, Egypt

It is currently believed that the morphology of dendrites depends on two main factors, namely the material of the cytoplasm and the path length of the signal before reaching the soma. In the present work the branching order is introduced as a third factor; it represents the variation of electrical resistivity of different dendritic segments. A mathematical modification of the optimum cost function is applied. The software package TREES toolbox is employed to reconstruct several types of cells, specifically LPTC, HSS, HSN and starburst amacrine. The effectiveness of the present analysis is assessed by both the electrotonic and Sholl footprints. The results indicate a higher degree of matching between the synthesized reconstructions of these cells and their original morphology. Moreover, a reduction in the storage memory of their in silico morphologies is achieved.

Keywords: Branching order, dendrites morphology, electrotonic footprint, nerve cells.

DENDRITES are the electric signal receivers of neuronal cells and have an important role in signal communication among neurons. They sprout in tree structures to enable connections with the axons of surrounding neurons, and have a computational role with respect to the postsynaptic potentials $^{1-4}$. Moreover, dendrite morphology has a crucial role in orientation selectivity of synaptic inputs ${ }^{5}$.

The relationship between neuron structure and its function is still a potent source of controversy. To study neuron morphology, two approaches are applied. First, dendrites and axons are reconstructed from experimental preparations. Neural tissues are stained so that their morphological fine details become accessible for inspection microscopically; they are then digitalized and stored. The most complete database is reported in NeuroMorpho.Org ${ }^{6}$. It reports a large number of neuron cells that are reconstructed from different laboratories and then standardized and classified. Secondly, neuron morphology is reconstructed by building models based on the rules governing their structure ${ }^{7-10}$. Cajal $^{11}$ proposed a hypothesis that describes roughly how the morphology of dendrites develops. He suggested that the shape of a dendritic tree should satisfy two requirements - basically minimization of both the building material (cytoplasm) and conduction time. Hence dendritic bifurcation is guided by minimiz-

*For correspondence. (e-mail: messiery30@gmail.com) ing what he called the cost function, which includes these two factors.

Cajal's hypothesis was implemented by Cuntz et al. ${ }^{10}$. They introduced a mathematical expression that comprises both the total wiring cost and path length cost. Accordingly, the total cost is given as

Total $\operatorname{cost}=$ Total wiring cost + bf $\times$ path length cost,

where total wiring cost is a function of the cytoplasm material as a conducting medium, path length cost is the Euclidian distance between an unconnected branching point and root of the tree, and bf is a balancing factor which is a number that weighs between these two costs and reflects the degree of contribution of each parameter to the total cost function. Hence Cuntz et al. ${ }^{10}$ introduced a model that implements the minimum spanning tree algorithm to reconstruct the dendritic tree.

Figure 1 illustrates their procedure to reconstruct the dendrite tree using actual nodal distribution. The validity of the reconstructed technique is assessed by comparing the original and synthesized distributions.

However, the importance of impedance variation in different segments has been recognized earlier by Van Pelt $^{12}$ and overlooked in previous dendrite reconstructing models. Since the branching segments increase in their cross-sectional areas as they approach the soma, the total resistance of each segment will have an important effect on transmitting the electric potential from the synapses to the soma. In the present work we introduce a modification to the total cost function by adding a third factor, which considers the change in electrical resistance due to bifurcations in dendritic structures. This adds an extra degree of freedom to determine the optimal reconstructed morphology.

We utilized the data available from previously reported neuronal tree morphology of some cells ${ }^{6}$. Our results

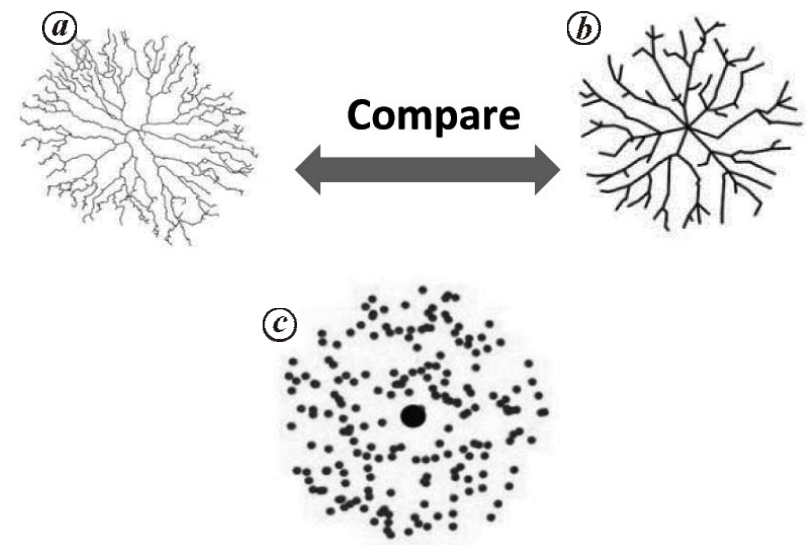

Figure 1. Methodology for reconstructing a dendritic tree. $\boldsymbol{a}$, An original dendritic tree. $\boldsymbol{b}$, The tree is reduced to a nodal distribution. $\boldsymbol{c}$, The produced synthetic tree from the proposed model. 
confirm that the branching order has a direct impact on generating synthesized trees that are electronically and geometrically comparable to the original morphology. Moreover, the transmission of electric current from the synapses to the soma can be calculated and analysed. Despite the fact that we take into consideration the application of cable theory, the present work could be extended to cover both the active and passive cases.

The present model examines the dependency of dendritic morphology on the spatio-electrical properties of dendrites as an additional term to Cajal's constraints. In the steady state, these properties are related to the change in input resistance of a dendritic segment as a factor affecting the bifurcation decision. Rall and Rinzel ${ }^{13}$ showed the importance of fine details in the neuronal structure; any small change in the neuronal morphology leads to a change in its electrical properties affecting the electrical output of a neural cell and hence its function.

The change in either the branching order or diameter of the branches leads to a significant variation in electric resistance. Hence the total axial resistance, membrane resistance and total membrane capacitance will be affected. Accordingly, either branching order or diameter of the branches reflects the change in electrotonic compartmentalization of dendritic trees. Since both factors are intercorrelated, graph theory was employed to select either one of them as a proposed third term in the cost function. It has been shown that branching order generates synthesized dendritic trees almost independent on the arbitrariness of the initial configurations ${ }^{14}$.

Hence, the proposed total cost function becomes

Total cost $=$ Total wiring cost $+b f \times$ path length cost

$+\mathrm{bw} \times$ branch order cost,

where branch order cost reflects the branching order at a tree node that could be connected to an unconnected one,

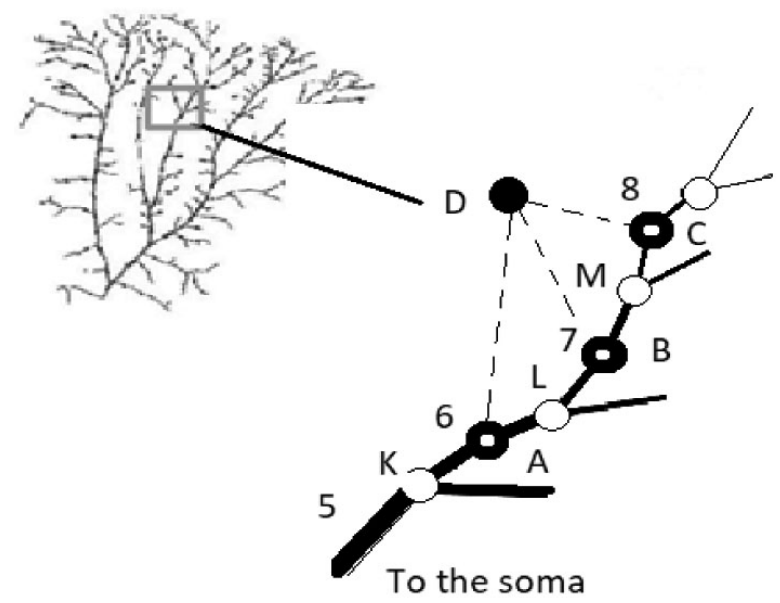

Figure 2. Node D chooses one of the unconnected target points A, B or $\mathrm{C}$ such that the total cost function is minimized. The numbers shown are examples of order parameters. and bw is the branching weight that provides the relative contribution of the branching order cost. The decisionmaking process based on eq. (2), is illustrated in Figure 2, which shows an arbitrary dendritic tree with an unconnected branching node $\mathrm{D}$. The increase in branch diameter is depicted as the dendrite approaches the soma. The numbers in Figure 2 represent the branching order of each segment. A minimum spanning tree algorithm begins to connect one node to another in the tree. Accordingly, node $\mathrm{D}$ may be connected to the tree via one of the three adjacent nodes: A, B or C. It is noteworthy that node D can connect only to the branching points where bifurcation may occur. Therefore, nodes K, L and M are ineligible for connections with node $\mathrm{D}$ because trifurcations are abnormal in neuronal morphologies. The most eligible connection must fulfil the optimal conditions of the total cost function. To elaborate, the distance DA is geometrically longer than both distances DB and DC; thus it contributes more to wiring cost. However, the connection through node $\mathrm{A}$ is the shortest to the soma than through nodes $\mathrm{B}$ or $\mathrm{C}$, and therefore DA has a better contribution to the path length due to less cytoplasm material. Moreover, connecting to node $\mathrm{A}$ has the smallest branching order (order 6 in the given example) than nodes B or C (orders 7 or 8 in the given example); thus less attenuation occurs to the signal. To achieve the optimal cost function bf and bw were adjusted by considering variations to their values from 0 to 1.0 .

The validity of the proposed synthesized morphologies was assessed by both the electrotonic and Sholl analyses. In the Cuntz model, a considerable number of synthesized morphologies are generated by changing bf until the optimal morphology is reconstructed. In the present work a method is introduced to reduce the electrotonic signature $^{10}$ and the Sholl analysis ${ }^{15}$ from their vector forms to

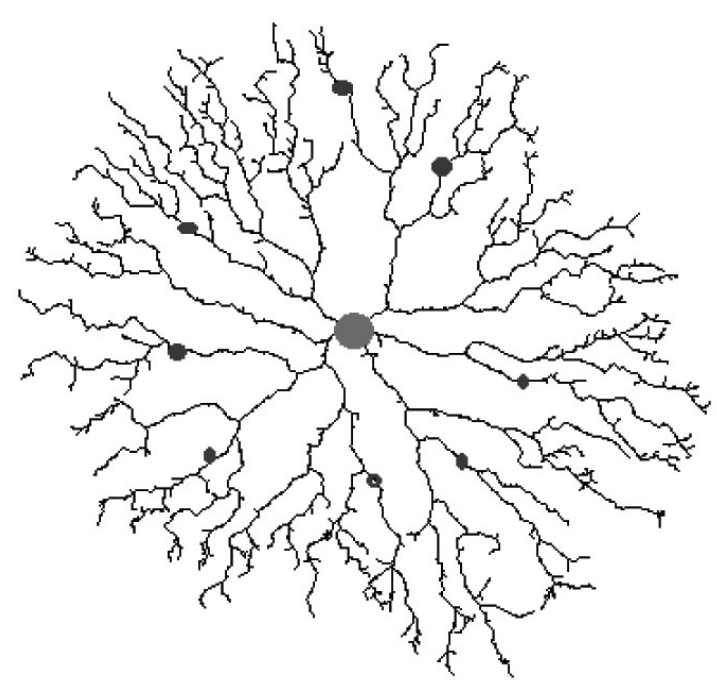

Figure 3. Arrangement of virtual electrodes (small dots) for calculating the electrotonic footprint for a starburst amacrine cell. The central large dot represents the soma. 

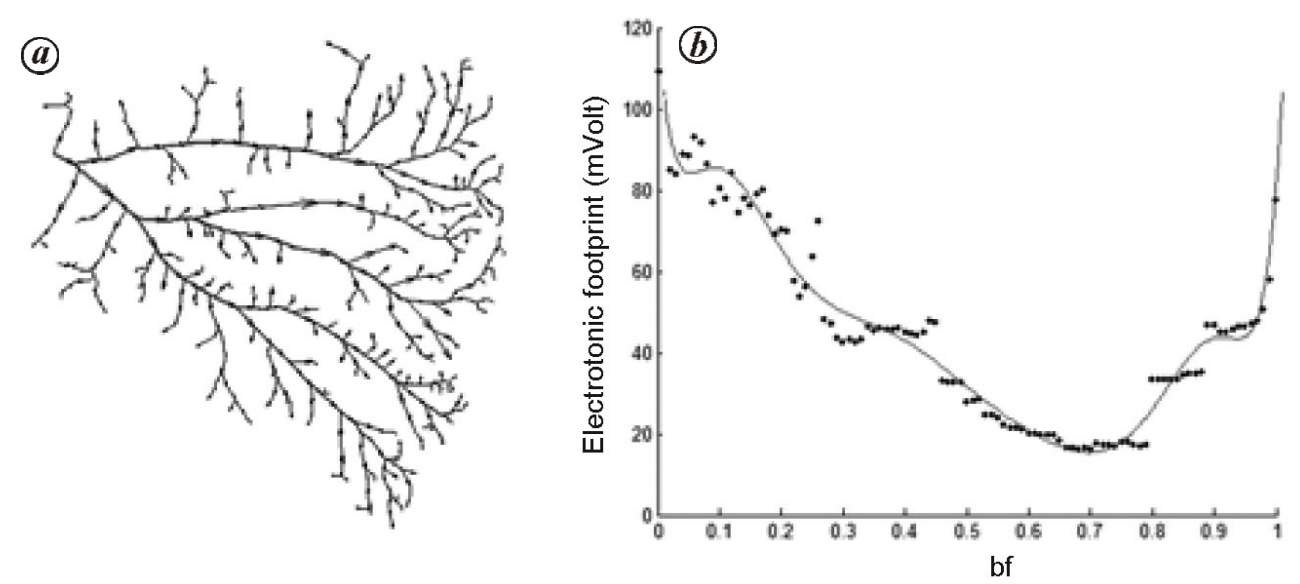

Figure 4. $\boldsymbol{a}$, The real reconstruction of a sample of flattened dendritic tree of LPTC. $\boldsymbol{b}$, The electrotonic footprint $(\mathrm{mV})$ versus the balancing factor $(\mathrm{bf})$.

scalar values. Hence, different synthesized neuronal cell morphologies may be readily compared, both electronically and geometrically.

The electrotonic signature is a matrix that calculates the steady-state electrotonic voltages at the nodes of the dendritic tree. Its calculation is necessary to evaluate neural morphologies from their electric characteristics perspective.

To compare a synthesized dendritic tree with the original one, Cuntz et al. ${ }^{10}$ developed the electrotonic signature as a measure of electric potential at the soma due to injecting a current in a single node. In the present work, this technique is extended to obtain the corresponding vector of the voltages at the soma due to injections at multiple nodes.

Figure 3 exemplifies the procedure followed to determine the electrotonic footprint of a dendritic morphology. $N$ electrodes at fixed places (represented by small dots) inject a virtual arbitrary constant current of $1 \mathrm{pA}$. The corresponding voltage $V(i)$ at the soma (represented by the central large dot) due to injection of an individual electrode $i$ is calculated. These voltages are stored as elements of a vector $V_{\text {synth }}$. On the other hand, voltage vector of the real reconstruction is obtained and stored in a vector $V_{\text {real }}$.

The average of absolute value of the difference between the two voltage vectors is calculated for the whole injection points and expressed by the following equation

$$
X(j)=\frac{1}{N} \sum_{i=1}^{N} \operatorname{abs}\left(V_{\text {real }}(i)-V_{\text {synth }_{j}}(i)\right),
$$

where $N$ is the total number of injection points and $X(j)$ represents the electrotonic footprint of one of the synthetic reconstructions of index $j$.

For $M$ of these synthetic reconstructions, eq. (3) is repeated using different values of bf and bw to calculate the resulting components of the $X(j)$ matrix. The minimum $X(j)$ is obtained; this value represents the optimal electrotonic match to the real reconstruction. Theoretically, the electrotonic footprint tends to zero when the two morphologies are identical.

Basically, the Sholl metric focuses on the geometrical aspects of neural morphologies ${ }^{15}$. A modified Sholl analysis is utilized to obtain scalar measures for the geometrical shape of a neuronal cell. For the real morphology of a neuron cell, the number of neuronal cell intersections with an imaginary Sholl sphere $i$ is stored in $\operatorname{Sholl}_{\text {real }}(i)$ vector. To elaborate, this vector expresses the number of intersections with concentric successive trees. A similar vector, $\operatorname{Sholl}_{\text {synth }}(i)$, is obtained for the synthetic structure of different values of bf and bw. The average of the absolute value of the difference between the Sholl vectors is calculated as

$$
Y(j)=\frac{1}{N} \sum_{i=1}^{N} \operatorname{abs}\left(\operatorname{Sholl}_{\text {real }}(i)-\operatorname{Sholl}_{\text {synth }_{j}}(i)\right),
$$

where $N$ is the total number of Sholl spheres, $j$ the index of one of the synthetic reconstructions and $y(j)$ represents the Sholl footprint. For $M$ synthetic reconstructions, eq. (4) is repeated and the minimum value is obtained. This value represents the optimal geometrical match to the real morphology. A full match is achieved when this minimum tends to zero.

We applied the electrotonic footprint measure on a sample of flattened dendritic tree of LPTC ${ }^{14}$ (Labula plate tangential cells of the blowfly visual system) and followed a modified technique of the TREES toolbox ${ }^{10}$.

Figure $4 a$ shows the real dendritic tree of this cell under study. In accordance with eq. (1), synthetic reconstructions were generated for different values of bf, where $0 \leq \mathrm{bf} \leq 1$ with a step interval of 0.01 . For each synthetic reconstruction, the electrotonic footprint was 


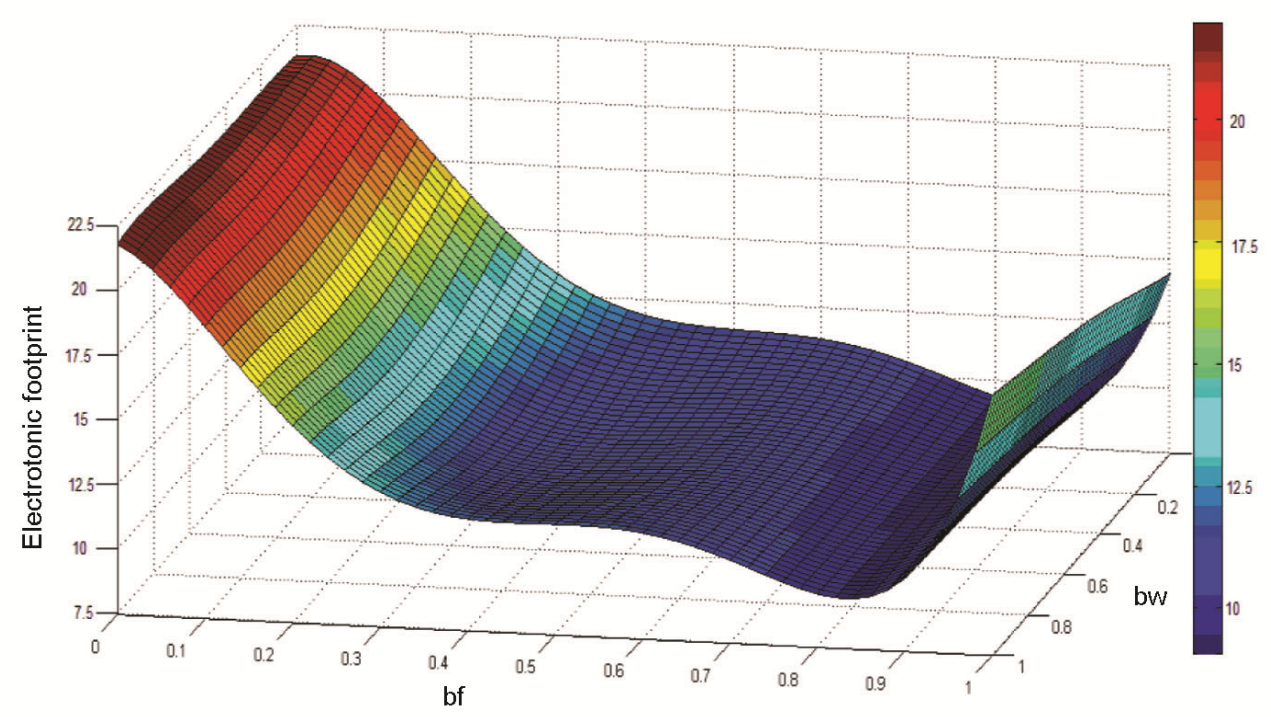

Figure 5. Plots of the electrotonic footprint $(\mathrm{mV})$ of the reconstruction of a sample of flattened dendritic tree of LPTC cell as a function of the two independent variables bf and balancing weight (bw). The optimal reconstruction is achieved when the footprint reaches a minimum.

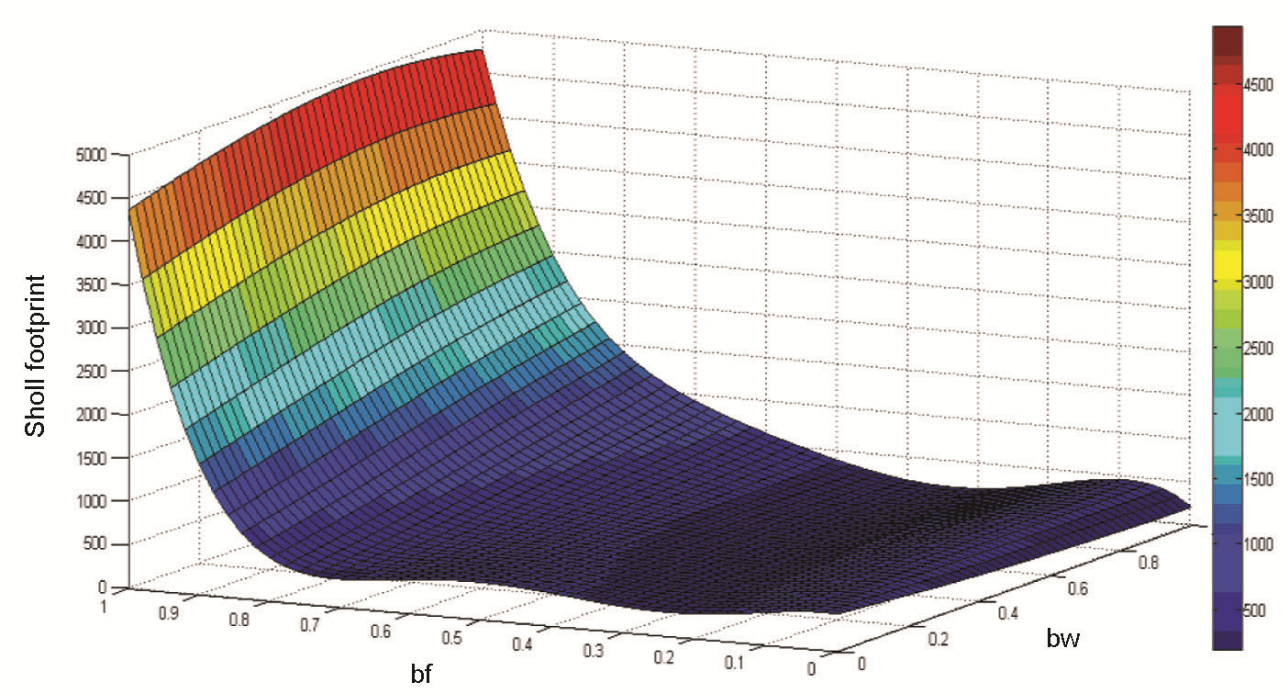

Figure 6. The Sholl footprint of reconstruction of a sample of flattened dendritic tree of LPTC by varying the two independent variables bf and bw from 0 to 1 .

calculated and plotted (Figure $4 \mathrm{~b}$ ). A minimum electrotonic footprint of $15.5 \mathrm{mV}$ was obtained at $\mathrm{bf}=0.71$, which resulted in the optimal synthesized tree.

On the other hand, the same sample was examined using the proposed model presented in eq. (2). The factors bf and bw were both varied from 0 to 1 in steps of 0.01 . The minimum spanning tree algorithm was applied. Figures 5 and 6 show the resulting electrotonic and Sholl footprints respectively. The optimal dendritic tree was generated when both the footprints reached their minimum values. Comparing the results obtained using eq. (1) with those of eq. (2) for the same sample, the calculations showed that minimum electrotonic footprint has dropped from 15.5 to $9.3 \mathrm{mV}$ using $\mathrm{bf}=0.823 \pm 0.107$ and $\mathrm{bw}=0.210 \pm 0.185$. Moreover, the $3 \mathrm{D}$ plot of the Sholl footprint (Figure 6) shows that it reaches an optimal value at $b f=0.866 \pm 0.021$ and $b w=0.394 \pm 0.005$. Using eq. (2) as opposed to eq. (1) has resulted in a drop in the Sholl footprint from 124 to 117 . Both the electrotonic and the Sholl technique produce consistent and close values for bf and bw. This indicates that the real dendritic morphology is better represented by the proposed model.

Figure $7 a$ shows the real LPTC as obtained from the Trees toolbox ${ }^{16}$. Figure $7 b$ shows the synthesized reconstruction using the present method while Figure $7 c$ 

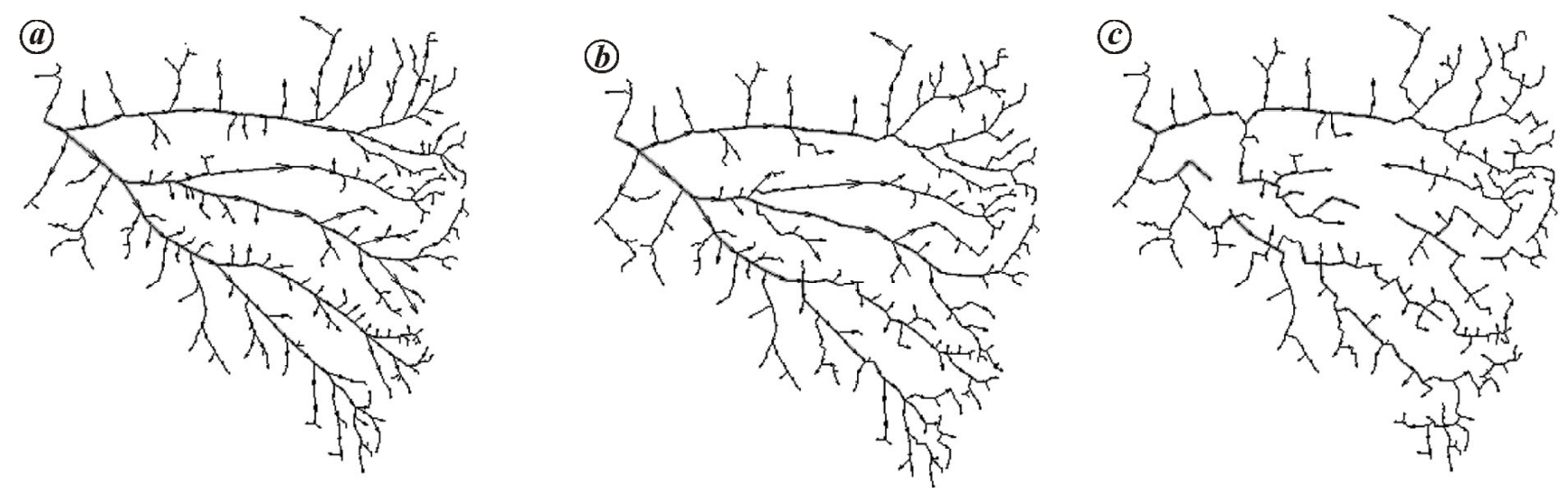

Figure 7. $\boldsymbol{a}$, The real reconstruction of a sample of flattened dendritic tree of LPTC. $\boldsymbol{b}$, The synthesized reconstruction using the present approach of both $\mathrm{bf}=0.866$ and $\mathrm{bw}=0.39 . c$, The synthesized reconstruction using eq. (1) for $\mathrm{bf}=0.45$.
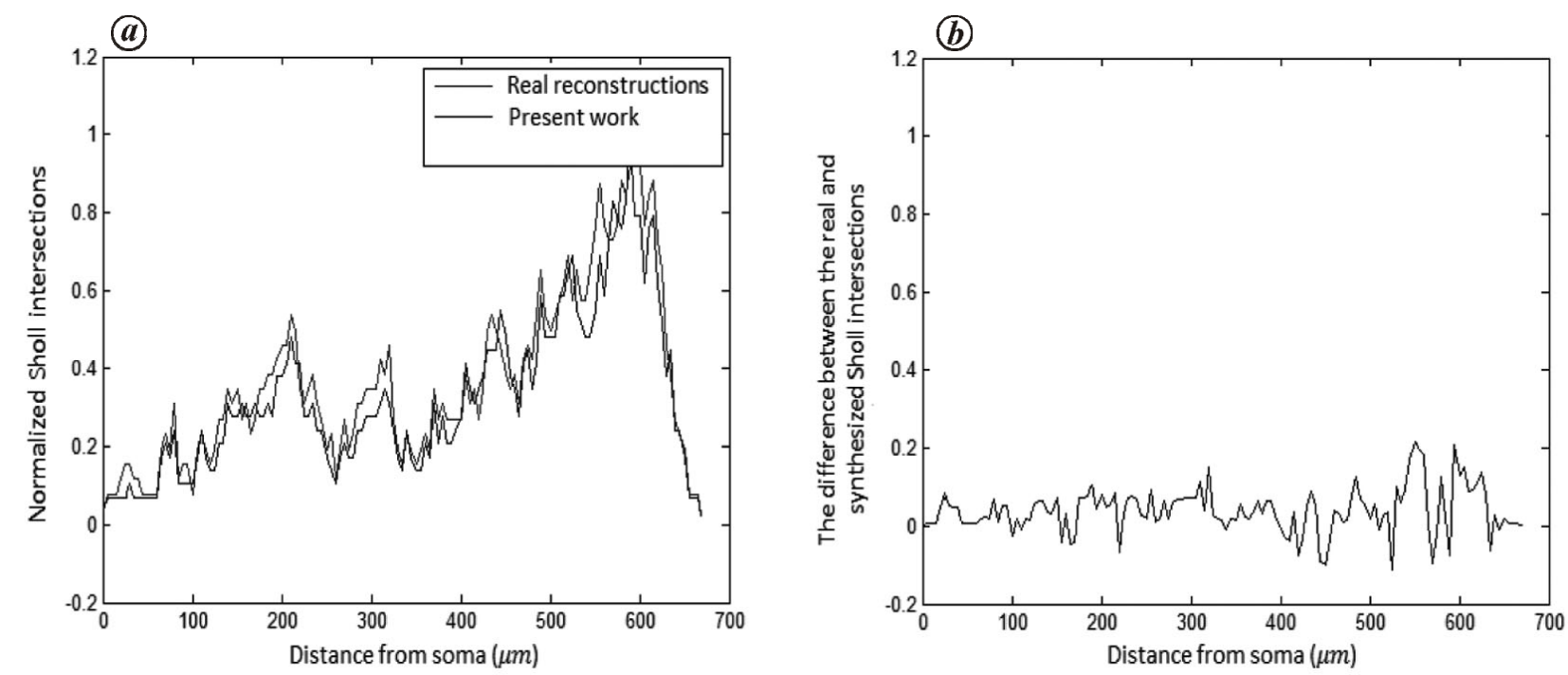

Figure 8. $\boldsymbol{a}$, The Sholl intersections of the real reconstruction (upper plot) of LPTC sample and optimal synthesized morphology (lower plot) based on eq. (2). b, The difference between the Sholl intersections of the real reconstruction and optimal synthesized tree using the present model.

shows a previously reported reconstruction ${ }^{10}$. By comparing Figure $7 b$ and $c$, it is clear that the present approach achieves better matching.

Figure $8 a$ compares the normalized Sholl intersections of real morphology of the LPTC sample and its optimally synthesized reconstruction using the present model; it shows a considerable match between the two. Figure $8 b$ shows the numerical difference between these Sholl patterns. The standard deviation of this difference is \pm 0.058 . On the other hand, this standard deviation jumps up to \pm 0.14 when the previous model is employed.

Further assessment of the present approach was achieved by applying the methodology to three other types of cells, namely HSS (Horizontal System of South location of a fly visual system), HSN (Horizontal System of North location of a fly visual system) and the starburst amacrine. The results are summarized in Table 1; it re- ports the optimal bf and bw values. The electrotonic and Sholl footprints of the previous and present work are presented as well.

In the present work, we have studied the effect of variation of cross-sectional area of dendrites on the transmission of electric signals from the synapses of a neural cell to the soma. In addition, reconstruction of synthetic morphologies helps determine the electric potential received at the soma when the synapses are activated.

The branching order is an important factor in determining the overall dendrite morphology. An extra parameter is added to the previously reported hypothesis to obtain a more realistic approach in which branching order is associated with decrease in the diameter of each branch starting from the soma to the synapses of a dendrite. Consequently, synthesized dendrite reconstructions become more convergent to their real neuronal structures. 
Table 1. Numerical values of bf and bw for optimal electrotonic and Sholl footprints for different cell types. The NeuroMorpho.Org id of each cell is also provided

\begin{tabular}{|c|c|c|c|c|}
\hline & & \multicolumn{3}{|c|}{ Cell } \\
\hline & & $\begin{array}{c}\text { HSS } \\
\text { NMO_01889 }\end{array}$ & $\begin{array}{c}\text { HSN } \\
\text { NMO_01889 }\end{array}$ & $\begin{array}{c}\text { Starburst amacrine } \\
\text { NMO_00765 }\end{array}$ \\
\hline \multirow[t]{2}{*}{ Optima electrotonic footprint } & bf & $0.05 \pm 0.00$ & $0.23 \pm 0.02$ & $0.30 \pm 0.01$ \\
\hline & bw & $0.38 \pm 0.02$ & $0.18 \pm 0.15$ & $0.58 \pm 0.03$ \\
\hline \multirow[t]{6}{*}{ Optimal Sholl footprint } & bf & $0.065 \pm 0.024$ & $0.15 \pm 0.01$ & $0.23 \pm 0.01$ \\
\hline & bw & $0.30 \pm 0.14$ & $0.73 \pm 0.17$ & $0.43 \pm 0.04$ \\
\hline & Present electrotonic footprint (mV) & 0.61 & $8.55 \times 10^{-4}$ & 1.95 \\
\hline & Previous electrotonic footprint (mV) & 0.74 & $12.9 \times 10^{-4}$ & 2.12 \\
\hline & Present Sholl footprint & 121 & 114 & 60 \\
\hline & Previous Sholl footprint & 129 & 133 & 66 \\
\hline
\end{tabular}

Moreover, the present work is aimed at introducing synthetic morphologies of several types of neuron cells. The reconstructions were examined using a modified scalar form of the electrotonic and Sholl matrices. Hence collective and precise measures of discrepancies between the original and synthetic morphologies are obtained. Accordingly, optimal values of bf and bw have been reported. Several cells are used and comparison is made between the present results and previous ones.

In addition, the present approach successfully demonstrates that in silico cell morphology can be generated only by five parameters, namely the optimum bf and bw factors, and the node coordinates $x, y, z$. Traditionally, digital storage of cell morphology requires at least seven parameters for each node ${ }^{17}$, specifically the node index; the region code; $x, y, z$ coordinates; diameter at the node location, and index of the parent node. Therefore, the storage space needed for neuronal cell morphology is reduced to almost two-third of the existing procedures, which enables researchers to handle a considerable number of cells.

1. Van Elburg, R. A. J. and Van Ooyen, A., Impact of dendritic size and dendritic topology on burst firing in pyramidal cells. PLoS Comput. Biol., 2010, 6(5), e1000781.

2. Mainen, Z. F. and Sejnowski, T. J., Influence of dendritic structure on firing pattern in model neocortical neurons. Nature, 1996, 382(6589), 363-366.

3. Silver, R. A., Neuronal arithmetic. Nature Rev. Neurosci., 2010, 11(7), 474-489.

4. Torben-Nielsen, B. and Stiefel, K. M., An inverse approach for elucidating dendritic function. Front. Comput. Neurosci., 2010, 4(128), 1-11.

5. Jia, H. et al., Dendritic organization of sensory input to cortical neurons in vivo. Neuroforum, 2010, 16(3), 236-237.

6. Ascoli, G. A., Donohue, D. E. and Halavi, M., NeuroMorpho.Org: a central resource for neuronal morphologies. J. Neurosci., 2007, 27(35), 9247-9251.

7. Ascoli, G. A., Progress and perspectives in computational neuroanatomy. Anat. Rec., 1999, 257(6), 195-207.

8. Ascoli, G. A., Krichmar, J. L., Scorcioni, R., Nasuto, S. J., Senft, S. L. and Krichmar, G. L., Computer generation and quantitative morphometric analysis of virtual neurons. Anat. Embryol., 2001, 204(4), 283-301.

9. Luczak, A., Spatial embedding of neuronal trees modeled by diffusive growth. J. Neurosci. Methods, 2006, 157(1), 132-141.

10. Cuntz, H., Forstner, F., Borst, H. and Häusser, M., One rule to grow them all: a general theory of neuronal branching and its practical application. PLoS Comput. Biol., 2010, 6(8), e1000877.

11. Cajal, S. R., The structure and connexions of neurons. Nobel Prize in Physiology or Medicine Lecture, Nobel Foundation, 1906, pp. 220-253.

12. Van Pelt, J., A simple vector implementation of the Laplacetransformed cable equations in passive dendritic trees. Biol. Cybern., 1992, 68(1), 15-21.

13. Rall, W. and Rinzel, J., Branch input resistance and steady attenuation for input to one branch of a dendritic neuron model. Biophys. $J ., 1973$, 13(7), 648-688.

14. Elias, M., A biophysics approach to generate synthetic morphologies for real dendritic reconstructions of neuronal cells, M Sc thesis, Cairo University, Egypt, 2015.

15. Sholl, D. A., Dendritic organization in the neurons of the visual and motor cortices of the cat. J. Anat., 1953, 87(Pt 4), 387-406.

16. Cuntz, H., Förstner, F., Borst, A. and Häusser, M., Trees toolbox. 2010, 90 .

17. Cuntz, H., Modelling dendrite shape from wiring principles. In The Computing Dendrite: From Structure to Function, Springer, 2014.

Received 19 April 2018; revised accepted 22 October 2018

doi: $10.18520 / \mathrm{cs} / \mathrm{v} 116 / \mathrm{i} 3 / 457-462$ 